\title{
Prediction of symptomatic improvement after exposure-based treatment for irritable bowel syndrome
}

Brjánn Ljótsson ${ }^{1 *}$, Erik Andersson ${ }^{2}$, Perjohan Lindfors ${ }^{3,4}$, Jeffrey M Lackner ${ }^{5}$, Karin Grönberg ${ }^{6}$, Katarina Molin ${ }^{6}$, Johanna Norén ${ }^{6}$, Karin Romberg ${ }^{6}$, Evelyn Andersson ${ }^{2}$, Timo Hursti ${ }^{6}$, Hugo Hesser $^{7}$ and Erik Hedman ${ }^{1,8}$

\begin{abstract}
Background: Several studies show that psychological treatments relieve symptoms for patients suffering from irritable bowel syndrome (IBS). However, there are no consistent findings that show what patient characteristics make a psychological treatment more or less likely to result in improvement. We have previously conducted a study of a newly developed internet-delivered cognitive behavioral therapy (ICBT) that emphasized exposure to IBS symptoms and IBS-related situations and reduced symptom-related avoidance. The study showed that the treatment led to improvement in IBS symptoms compared to a waiting list and that treatment gains were maintained over a 15-18 month follow-up period. The aim of the present study was to investigate several possible predictors of short- and long-term treatment outcome in terms of symptom improvement, based on data collected in the previously conducted treatment trial.
\end{abstract}

Methods: Demographics, comorbid psychological distress, IBS-related fear and avoidance behaviors, and IBS-related disability were investigated as predictors of treatment outcome in the sample consisting of 79 participants diagnosed with IBS who had undergone 10 weeks of ICBT. Predictors that were significantly correlated with symptom levels at post-treatment and follow-up were entered into multiple regression analyses that controlled for pre-treatment symptom levels.

Results: There were measures within each domain, i.e., comorbid psychological distress, IBS-related fear and avoidance behaviors, and IBS-related disability, with the exception of demographic data, that were correlated with the symptom levels at post-treatment and follow-up. However, when these were entered into a multiple regression analyses that controlled for pre-treatment levels, none remained a significant predictor of the post-treatment and follow-up symptomatic status.

Conclusions: The study did not find any individual characteristics that made patients more or less likely to respond to the exposure-based ICBT. The finding that comorbid psychological distress did not predict outcome is in accordance with previous studies. Reliable predictors for response to any type of psychological treatment for IBS remain to be established.

Keywords: Irritable bowel syndrome, Exposure, Internet, Cognitive behavior therapy, Psychological treatment, Prediction analysis

\footnotetext{
* Correspondence: brjann.ljotsson@ki.se

'Department of Clinical Neuroscience, Division of Psychology, Karolinska

Institutet, Nobels väg 9, Stockholm 171 65, Sweden

Full list of author information is available at the end of the article
} 


\section{Background}

Irritable bowel syndrome (IBS) affects about $11 \%$ of the population [1], is characterized by recurring abdominal pain and diarrhea and/or constipation [2] and associated with decreased quality of life [3] and increased health-care consumption [4]. Several different types of psychological treatments have been studied as interventions for IBS, including cognitive behavior therapy (CBT), psychodynamic therapy, and hypnotherapy [5]. These treatments generally show beneficial effects but treatment effects vary considerably between studies of the same treatment types [6], and little is known about what patient characteristics predict successful outcome from psychological treatment. More knowledge in this regard is important as it can contribute to a better matching of patients to treatments and could ultimately be used to increase the total proportion of participants who respond to treatment [7].

The observation that many patients with IBS present with comorbid psychiatric conditions or psychological distress [8], which are associated with increased symptom severity [9], is often used as rationale for the application of psychological treatments for IBS [6]. Based on this rationale for psychological treatment, it would be reasonable to assume that presence of psychiatric comorbidity or high psychological distress would imply suitability, i.e., better treatment response, for psychological interventions. However, results from prediction analyses have been contradictory. We have found four studies that reported a positive association between psychological distress at pretreatment and post-treatment improvement [10-13]. Eight studies have reported the reverse association, i.e., that psychological distress predicted worse outcome [14-21]. Finally, three studies reported no association between psychological distress and outcome [22-24]. The measures of psychological distress and the predicted outcome have differed between studies (not all have used symptomatic improvement as outcome), and the studies may therefore not be completely comparable. Notwithstanding the different methods, there does not seem to be much data backing up the common proposition that patients with IBS should benefit from psychological treatments just because they display psychological distress e.g., [5].

However, although most psychological treatments for IBS, in accordance with the common rationale, target comorbid psychological distress [6], there are also cognitive behavioral treatments that target disorder-specific behavioral patterns in IBS. During the last decade, several studies of CBT based on behavioral exposure have been conducted where the primary target of treatment has been IBS-related avoidance and worry rather than comorbid psychological distress [25-30]. This behavioral pattern, often referred to as gastrointestinal symptom-specific anxiety (GSA) [31], is associated with increased symptom severity and decreased quality of life $[32,33]$. The exposure-based treatments have consistently produced large and stable effect sizes on measures of IBS symptoms and quality of life [25-30]. Thus, the treatments that target GSA seem to be an exception from the rule that psychological treatments for IBS show large between-study variation in treatment effects. Based on the model employed in these treatments targeting GSA, it would be reasonable to assume that patients with IBS who show high levels of GSA, more specifically IBS-related avoidance behaviors, worry about IBS symptoms, and disability related to IBS, could be suitable candidates for treatments targeting this symptom-specific pattern. We are only aware of one study that has investigated the predictive value of GSA and disability on outcome after an exposurebased treatment. This study included a consecutively recruited sample of clinical patients with IBS and did not find that high pre-treatment levels of GSA and disability were related to larger improvement in IBS symptoms after an internet-delivered cognitive behavioral treatment (ICBT) based on exposure exercises [25]. However, these analyses were based on a small sample with a large proportion of missing data, as 7 of $30(23 \%)$ participants in the treatment arm did not complete the post-treatment data, limiting the statistical power to detect meaningful associations and possibly introducing bias in the prediction analyses. Another previous trial of ICBT for IBS [27], had less missing data and several possible predictors were measured before treatment, including psychiatric comorbidity, GSA, and disability. Furthermore, the entire sample was assessed at a long-term follow-up [34], allowing for prediction of both the short- and long-term treatment effects. The present predictor study is based on data from that trial.

The aim of the present study was to investigate the role of psychological distress, GSA and IBS-related disability as predictors of short- and long-term symptomatic improvement following treatment with ICBT for IBS. Based on the inconsistent findings in the IBS research on outcome prediction we did not formulate any specific hypotheses.

\section{Methods}

\section{Design}

This study included 79 self-referred participants with a diagnosis of IBS who had participated in a randomized controlled study (ClinicalTrials.gov: NCT01171053) of ICBT for IBS, where they were randomized to either treatment $(n=39)$ or waiting list control $(n=40)$ for 10 weeks ([27]. After the 10-week post-treatment assessment participants on the waiting list were crossed over to receive treatment and subsequently completed a post-treatment assessment after 10 additional weeks. In addition, both groups participated in a long-term follow-up assessment [34]. Since both groups were followed up simultaneously, the time between treatment completion and follow-up assessment was 18 months for the treatment group and 15 months for the group who received treatment after 
having been on the waiting list. In the present study, the two samples were pooled together to predict the symptomatic changes from pre-treatment to post-treatment and from pre-treatment to follow-up, based on pre-treatment characteristics. This study was approved by the Regional ethical review board in Stockholm (No: 2007/1451 - 31/4).

\section{Participants}

All participants had received a clinical diagnosis of IBS before inclusion and fulfillment of Rome III criteria [2] was confirmed before inclusion. Inclusion criteria, recruitment procedure and participant characteristics are detailed in the original report [27]. Because 6 of 85 participants in the original trial withdrew from the study before the post-treatment assessment, this study included the remaining 79 (93\%) who had participated in the post-treatment $(n=76)$ and/or the follow-up assessment $(n=74)$, and thus provided improvement data for analysis in the present study. Sample characteristics are presented in Table 1.

\section{Measures}

All self-report measurements in the study were completed online. Online assessment is considered a well-validated form of administration of self-assessments and has been shown to produce results similar to pen-and-paper administration [35].

\section{Outcome}

The Gastrointestinal Symptom Rating Scale -IBS version (GSRS-IBS) [36] was used as the outcome measure in this study. Because IBS symptoms vary considerably over time, the GSRS-IBS was administered weekly during three weeks at pre-treatment and post-treatment and four weeks at follow-up. The weekly average score at each assessment point was used to get reliable estimates of the participants' symptom levels.

\section{Measures of psychological distress}

During the inclusion procedure in the original study all participants underwent psychiatric diagnostic assessment

Table 1 Pre-treatment characteristics and correlations with outcome variables

\begin{tabular}{|c|c|c|c|c|c|}
\hline & \multirow[b]{2}{*}{$n$} & \multirow[b]{2}{*}{$m$} & \multirow[b]{2}{*}{ sd } & \multicolumn{2}{|c|}{ Correlation with GSRS-IBS } \\
\hline & & & & Post-treatment & Follow-up \\
\hline \multicolumn{6}{|l|}{ Outcome variables } \\
\hline GSRS-IBS at post-treatment & & 35.18 & 13.77 & & $.53^{*}$ \\
\hline GSRS-IBS at follow-up & & 32.58 & 12.71 & $.53^{*}$ & \\
\hline \multicolumn{6}{|l|}{ Demographic variables } \\
\hline Sex (male/female) & $67 / 12$ & & & .14 & -.17 \\
\hline Age & & 34.25 & 9.25 & -.05 & .03 \\
\hline Duration of IBS & & 13.70 & 11.10 & .06 & .07 \\
\hline University studies (yes/no) & $52 / 27$ & & & -.19 & -.04 \\
\hline \multicolumn{6}{|l|}{ Predictor variables } \\
\hline GSRS-IBS at pre-treatment & & 47.74 & 11.00 & $.50^{*}$ & $.30^{*}$ \\
\hline Study group (treatment/waiting list) & $39 / 40$ & & & -.20 & -.05 \\
\hline Any anxiety disorder (yes/no) & $39 / 40$ & & & .15 & .16 \\
\hline Major depressive disorder (yes/no) & $15 / 62$ & & & .12 & $.27^{*}$ \\
\hline MADRS-S & & 11.20 & 7.99 & .20 & $.36^{*}$ \\
\hline SSP-PSTA & & 2.56 & 0.69 & .07 & .17 \\
\hline SSP-STA & & 2.52 & 0.58 & .03 & .03 \\
\hline SCL-SOM & & 13.52 & 9.19 & .17 & .18 \\
\hline VSI & & 42.92 & 17.42 & .21 & $.28^{*}$ \\
\hline CSQ-CAT & & 14.72 & 7.78 & $.35^{*}$ & $.32^{*}$ \\
\hline ASI & & 19.24 & 12.01 & $.25^{*}$ & .17 \\
\hline IBS-QOL & & 52.45 & 20.57 & $-.41^{*}$ & $-.31^{*}$ \\
\hline SDS & & 12.13 & 8.28 & $.37^{*}$ & $.26^{*}$ \\
\hline
\end{tabular}


using the Mini-International Neuropsychiatric Interview (MINI) [37]. The MINI is a structured diagnostic interview that identifies DSM-IV Axis-I disorders. The interviews were performed by advanced graduate students in their final year of the Swedish psychology program and a clinical psychologist. All interviewers were trained in using the MINI and diagnosing psychiatric disorders.

The Montgomery Åsberg Depression Rating Scale - Self report (MADRS-S) [38] was used to measure depressive symptoms. The somatic trait anxiety (SSP-STA) and psychic trait anxiety (SSP-PSTA) are two subscales of Swedish Universities Scales of Personalities [39] and were used to measure tendencies to feeling tense, restless, and worried. To measure somatization, i.e., the tendency to experience somatic symptoms in response to psychological distress, we used the somatization subscale of the Symptom Checklist90 (SCL-SOM) [40]. The SCL-SOM measures the severity of different somatic symptoms during the last week, e.g., headache, dizziness, pain, nausea, and weakness.

\section{Measures of GSA and related constructs}

The Visceral Sensitivity Index (VSI) [31], was used to measure GSA, i.e., heightened anxiety and worry about visceral sensation and IBS-associated situation (such as not being near a toilet) and behavioral attempts to avoid these stimuli. We used the catastrophizing subscale of the Coping Strategies Questionnaire, which measures the tendency to perceive pain sensation as catastrophic and unbearable (CSQ-CAT) [41]. In the present study, the participants were instructed to report their reactions also to other IBS symptoms such as constipation, diarrhea, and bloating, in addition to pain. The Anxiety Sensitivity Index (ASI) [42] was used to measure the degree to which participants believed that symptoms of anxiety and arousal could have negative consequences, such as illness, embarrassment, or increased anxiety.

\section{Measures of disability}

The Irritable Bowel Syndrome Quality of Life Instrument (IBS-QOL) [43] was used to assess the impact on quality of life for patients with IBS in several domains, including symptom interference with activity and impact on relationships. The Sheehan Disability Scales (SDS) [44] were used to assess symptom induced disability in three domains: social, work, and family.

\section{Treatment}

The treatment consisted of a 10-week ICBT-protocol based on exposure and mindfulness exercises. Numerous studies have demonstrated that cognitive behavioral treatments for many somatic and psychiatric disorders can be delivered over internet with effects similar to face-to-face treatment [45]. The ICBT protocol in the present study was mainly based on the principle of behavioral exposure, i.e., participants provoked IBS symptoms and reduced their avoidance of stimuli that induced symptoms or worry about symptoms. The proposed mechanism was that the exposure exercises, aided by mindfulness exercises, would break the cycle of symptom and disability maintenance that the GSA behavioral pattern constitutes, leading to improvement in symptoms and quality of life. The original report contains a detailed description of the treatment program and results [27].

\section{Assessment}

All self-assessments were collected before treatment start. The GSRS-IBS was also completed at post-treatment and 15-18 months follow-up. The telephone interviews, SSPSTA, and SSP-PSTA were administered at the screening phase of the study, i.e. 1 week to 3 months before treatment start for the original treatment group and about 36 months before treatment start for the waiting list.

\section{Analysis}

All statistical analyses were performed using SPSS 21. The two samples, one with participants who received ICBT directly and the other comprising participants who received ICBT after 10 weeks on waiting list, were pooled into a single sample to increase statistical power. This was deemed acceptable, as there were no statistically significant differences between the groups on any of the demographics (age, sex, and duration of IBS), predictors, or dependent variables, i.e., the post-treatment and follow-up scores on the GSRS-IBS. The analyses were conducted in two steps. First, the GSRS-IBS post-treatment and followup scores were correlated with the demographic and predictor variables. Second, all demographic and predictor variables that were significantly correlated with the posttreatment or follow-up GSRS-IBS scores were entered into two separate multiple linear regression models, using the post-treatment and the follow-up GSRS-IBS scores as the dependent variable. The GSRS-IBS pre-treatment score was included in both regression models to adjust for initial baseline level and account for reliability of the measurement. This procedure often yields results equivalent to using residualized change scores in the prediction of treatment outcomes [46] and is superior to dichomotization (such as median split) in terms of increased power and decreased risk of type-I errors [46,47]. We also examined the impact of outliers and multicollinearity on the results. The power of the analysis to detect a predictor variable with a moderate effect sixe $\left(f^{2}=.15\right.$ or higher $)$ was $93 \%$ with alpha-level set at 05 .

\section{Results}

\section{Descriptive statistics}

Table 1 displays descriptive statistics of the demographic, predictor, and outcome variables. As can be seen in 
Table 1, the mean GSRS-IBS score decreased from 47.74 at pre-treatment to 35.18 at post-treatment and 32.58 at follow-up for the whole sample. According to the MINI interview before randomization, 42 participants had diagnosable psychiatric condition, 15 participants were diagnosed with major depressive disorder and 39 participants with an anxiety disorder (the most common were agoraphobia, $n=21$, generalized anxiety disorder, $n=18$, social anxiety disorder, $n=10$, and panic disorder, $n=10$ ).

\section{Bivariate correlations}

The bivariate correlations between the demographic/predictor variables and the outcome measures are displayed in Table 1. The pre-treatment scores on the GSRS-IBS were significantly correlated with both the post-treatment and follow-up scores on the same measure. In addition, the CSQ-CAT, ASI, IBS-QOL, and SDS were significantly correlated with the post-treatment GSRS-IBS score and the diagnosis of major depressive disorder as well as scores on the MADRS-S, VSI, CSQ-CAT, IBS-QOL, and SDS were significantly correlated with the follow-up GSRS-IBS score. With the exception of IBS-QOL, all significant correlations were positive. This means that more severe symptoms or disability were correlated with worse outcome on the GSRS-IBS. None of the demographic variables were related to outcome.

\section{Regression analyses}

All predictors that were significantly correlated with the outcome were entered into multiple linear regression analyses, separate for the post-treatment and follow-up scores of the GSRS-IBS. The results of the regression analyses are presented in Table 2 . None of the predictor variables remained significantly associated with the post-treatment or follow-up scores on the GSRS-IBS, with the exception of the pre-treatment GSRS-IBS score, which significantly predicted the post-treatment GSRS-IBS score. The models accounted for 28.7\% $(F(5,70)=5.64$, $\mathrm{p}<.001)$ and $19.5 \% \quad(\mathrm{~F}(7,66)=2.28, \mathrm{p}=.039) \quad$ of the variances in GSRS-IBS post-treatment and follow-up scores, respectively.

Several alternative models were evaluated in sensitivity analyses. (1) When testing for possible multicollinearity, the GSRS-IBS and VSI scores were found to be highly correlated, $r=.84$. Because the model predicting follow-up scores included both these measures, alternative models were tested with either the VSI or IBSQOL removed. (2) One outlier who scored more than 3 standard deviations above the sample mean on the GSRS-IBS at both post-treatment and follow-up was identified, and all analyses were rerun without this outlier. (3) The analyses were also rerun separately for the original study's treatment group and waiting list group. (4) Each of the predictors that showed a significant bivariate correlation with the outcome measures were investigated separately by entering them into regression models that only controlled for the pre-treatment GSRS-IBS score (i.e., no other predictors were entered into the model). None of these alternative models gave substantially different results.

Table 2 Regression models predicting GSRS-IBS scores at post-treatment and follow-up

\begin{tabular}{|c|c|c|c|c|c|c|c|}
\hline & Coeff & $\mathrm{Cl}-$ & $\mathrm{Cl}+$ & $\beta$ & $t$ & $p$ & Partial $r$ \\
\hline \multicolumn{8}{|c|}{ Prediction of GSRS-IBS at post-treatment. $R=.536 . R^{2}=.287 . p<.001$} \\
\hline Intercept & 10.66 & -18.52 & 39.84 & & 0.73 & .469 & \\
\hline GSRS-IBS pre-treatment & 0.51 & 0.19 & 0.83 & .40 & 3.18 & .002 & .36 \\
\hline CSQ-CAT & -0.03 & -0.58 & 0.51 & -.02 & -0.10 & .921 & -.01 \\
\hline ASI & -0.01 & -0.29 & 0.27 & -.01 & -0.08 & .939 & -.01 \\
\hline IBS-QOL & -0.05 & -31.64 & 21.48 & -.08 & -0.38 & .704 & -.05 \\
\hline SDS & 0.27 & -0.23 & 0.75 & .16 & 1.08 & .284 & .13 \\
\hline \multicolumn{8}{|c|}{ Prediction of GSRS-IBS at follow-up. $R=.441 . R^{2}=.195 . p=.039$} \\
\hline Intercept & 12.75 & -25.75 & 51.26 & & 0.66 & .511 & \\
\hline GSRS-IBS pre-treatment & 0.21 & -0.12 & 0.54 & .18 & 1.25 & .215 & .15 \\
\hline Major depressive disorder & 5.31 & -2.32 & 12.94 & .17 & 1.39 & .169 & .17 \\
\hline MADRS-S & 0.32 & -0.15 & 0.78 & .20 & 1.37 & .177 & .17 \\
\hline VSI & 0.08 & -0.22 & 0.38 & .11 & 0.53 & .597 & .07 \\
\hline CSQ-CAT & -0.01 & -0.59 & 0.58 & .00 & -0.02 & .985 & .00 \\
\hline IBS-QOL & 0.03 & -30.83 & 36.68 & .05 & 0.17 & .863 & .02 \\
\hline SDS & 0.04 & -0.46 & 0.54 & .03 & 0.17 & .866 & .02 \\
\hline
\end{tabular}

Cl-: $95 \%$ Confidence interval, lower bound. $\mathrm{Cl}+95 \%$ Confidence Interval, upper bound. GSRS-IBS Gastrointestinal Symptom Rating Scale, IBS version, MADRS-S Montgomery Åsberg Depression Rating Scale - Self Report, VSI Visceral Sensitivity Index, ASI Anxiety Sensitivity Index, CSQ-CAT Coping Strategies Questionnaire Catastrophizing subscale, IBS-QOL Irritable Bowel Syndrome Quality of Life Instrument, SDS Sheehan Disability Scales. 


\section{Discussion}

In this study, we aimed to investigate possible predictors of symptomatic improvement in a previously conducted trial in which study participants with IBS underwent ICBT, which targeted GSA by exposure exercises and mindfulness training. The investigated predictor domains were psychological distress, GSA, and disability. The original reports showed that the ICBT was effective in reducing IBS symptoms and that improvements were maintained over a long follow-up time $[27,34]$. Although several of the prognostic factors were correlated with the symptomatic outcome, none remained a significant predictor when entered into a multiple regression model that included the pretreatment symptom levels. Thus, the results in this study largely follow the same pattern of previous prediction studies in the IBS field, with no variable appearing as a clear and consistent predictor of treatment outcome. This is important information from a clinical perspective as it means that the clinician could, considering that ICBT leads to symptom reduction, offer the treatment regardless if the patient is an older woman with comorbid anxiety and severe IBS symptoms or a younger man without psychiatric comorbidity and moderate IBS symptoms.

Because the treatment under investigation in this study did not target comorbid psychological distress, we did not expect the presence of comorbid psychological distress to be predictive of treatment success. However, it is interesting to note that the proposed maintaining mechanisms of the psychiatric disorders found in this sample (primarily depression, panic disorder, social anxiety disorder, and generalized anxiety disorder) are similar to how GSA has been suggested to maintain IBS. Cognitive behavioral models emphasize how threat-orientation, worry, and avoidance related to disorder-specific stimuli serve to maintain these disorders e.g., [48]. Given the high comorbidity between common psychiatric disorders, this behavioral pattern has been suggested to be a shared causal factor between many psychiatric disorders, labeled emotional or experiential avoidance [49]. Similarly, there is a large overlap between IBS and other functional somatic disorders, e.g., fibromyalgia, chronic pain, and chronic fatigue syndrome [8], and worry, fear, hypervigilance, and avoidance in response to the disorder-specific symptoms have also been suggested to be important maintaining factors in these disorders as well as IBS [50]. Thus, emotional or experiential avoidance seems to be a behavioral phenomenon that unifies these functional somatic and psychiatric disorders and GSA may simply be a manifestation of this behavioral pattern in the context of IBS. Therefore, a patient who presents with both depression and IBS could be said not to be suffering from two comorbid disorders, of which one is especially suitable for psychological treatment, but rather to be displaying an avoidant behavioral pattern in response both to bowel symptoms and the routine demands of life, while a patient with IBS but without psychiatric comorbidity only displays this behavioral pattern in response to bowel symptoms. In essence, this putative explanation of the psychiatric comorbidity between the IBS and psychiatric disorders does not stipulate that psychiatric disorders should be present or absent for a GSA-focused treatment to work for IBS.

We have recently demonstrated that change on the VSI is a mediator of treatment effect on IBS symptoms in the exposure-based treatment for IBS [6]. This finding is in line with the proposed role of GSA as a maintaining factor in IBS and it could therefore be expected that patients who score high on the VSI would benefit more from the treatment than patients with low VSI scores. However, we did not find that pre-treatment scores on the VSI predicted symptomatic improvement. The stable placebo response in IBS may be a possible explanation for this finding. Reviews have shown that about $40 \%$ of patients with IBS respond to placebo treatments [51]. It could be hypothesized that even if participants with high levels of GSA, as measured by the VSI, responded favorably to the exposure exercises, participants with lower levels of GSA could also show treatment response, but this response would be due to unspecific factors rather than the specific interventions in the treatment. Even if the placebo-induced treatment response among low-GSA participants was smaller than the interventionspecific response for high-GSA participants, these two "competing" mechanisms of treatment response would mean reduced power to detect the influence of pretreatment GSA levels on symptomatic reduction.

This reasoning is partly supported by our recent study of mechanisms of change. There we observed that in the exposure treatment, engagement in exposure and mindfulness exercises was associated with improvement on the VSI (which in turn was associated with improvement on the GSRS-IBS) but in the comparison arm, a stress management condition, engagement in strategies such as relaxation and lifestyle changes was not associated with change in any measured mechanism [6]. Thus, the effect in the stress management arm may have been due to non-specific factors rather than specific symptom maintaining factors in IBS. Also, it is important to underscore that it is not necessary for GSA to predict outcome even if it mediates the treatment effect. It could be that reducing GSA leads to decreased symptoms, but that participants make similar GSA reductions, regardless of initial baseline levels of GSA. If this was the case, than there would be no strong predictive effect of GSA on outcome as assessed in the present study.

There were some important limitations that need to be considered when interpreting the results from this study. First, this was a secondary analysis on data from a study that was not designed to address the questions posed in this article. The lack of predictive value of the 
measures may be due to the nature of the sample where all participants were self-referred and therefore prepared to engage in a psychological treatment. A larger and more representative sample could have presented with a larger variation in pre-treatment characteristics and outcome and thus revealed true differences between low and high scoring groups that were not detectable in the sample under investigation. Second, a larger sample could also have provided more power to detect meaningful effects of the pre-treatment characteristics on the GSRS-IBS at post-treatment and follow-up. For example, the difference between depressed and non-depressed participants on the GSRS-IBS follow-up score was 5.31 with a $95 \%$ confidence interval of -2.32 to $12.94(p=.169)$, controlling for all other predictors. As the follow-up score on the GSRS-IBS was 32.58 , the non-significant difference of 5.31 points between depressed and non-depressed could actually represent a meaningful difference in outcome (especially when considering that by convention, the GSRS-IBS is scored between 13 and 91, rather than between 0 and 78, which in essence means that all difference scores should be compared with absolute scores subtracted with 13, i.e., a difference of 5.31 compared with a zero-based mean follow-up score of 19.58). Third, most of the psychiatric assessments were made by final year psychology students who assessed the presence of psychiatric diagnosis in interviews conducted over the telephone. The proportion of participants with a psychiatric disorder in this study, 42 of 79 (53\%), is close to the common approximation that about half of the IBS population has a diagnosable psychiatric disorder [52]. Still, with inexperienced assessors, individual participants may have been misclassified, limiting the validity of the prediction analyses based on the presence of psychiatric diagnosis. Fourth, the VSI may not be a measure that adequately captures the GSA phenomenon as it pertains to the exposure-based treatment. Of the 13 items on the VSI, only two measure behavioral responses to the IBS in terms of avoidance and safety behaviors while the remaining 11 items measure symptom-related worry, preoccupation, and sensitivity [31]. Because the exposure-based treatment mainly targets excessive avoidance and control behaviors, the VSI may not measure the behavioral aspects of GSA that are most relevant for prediction of outcome of this treatment. The Irritable Bowel Syndrome-Behavioral Responses Questionnaire (IBS-BRQ) [53], which only measures IBS-related avoidance and control behaviors, could have proved to be a better measure of suitability for the treatment. In fact, high scores on the IBS-BRQ have been shown to predict better response to CBT for IBS [13]. However, that specific CBT protocol was not exposure-based [54] and the predicted outcomes were work and social adjustment and not symptomatic improvement. Therefore, the results from the prediction study that included the IBSBRQ may not be comparable with this study.
Given the results of this and previous prediction studies, it is possible that the search for individual characteristics as stable predictors of treatment outcome is futile. It is also important to note that even if variation in specific individual characteristics were found to be associated with treatment outcome, most likely they will be associated with an increased likelihood that symptomatic improvement will occur rather than be binary determinants of whether an individual participant will respond or not. An important venue of future research could then be to focus on other potential predictors of outcome related to the therapeutic process rather than pre-treatment characteristics, for example treatment expectancy, treatment adherence, and treatment response patterns. The outcome of ICBT for fibromyalgia, social anxiety disorder, severe health anxiety disorder has been shown to be associated with the participants' adherence to treatment [55-57] and early response during the course of face-to-face CBT for IBS has been associated with better long-term improvement [58].

\section{Conclusions}

The findings of the present study suggest that ICBT for IBS has similar effects on symptoms regardless of demographic characteristics and psychiatric comorbidity in a self-referred sample. Considering the large prevalence of IBS and the many different psychological treatment protocols that have been developed, the need for more knowledge about how to match patients to treatment remains.

\section{Abbreviations}

ASI: Anxiety sensitivity index; CBT: Cognitive behavior therapy; CSQ-CAT: Coping strategies questionnaire - catastrophizing subscale; GSA: Gastrointestinal symptom-specific anxiety; GSRS-IBS: Gastrointestinal symptom rating scale - IBS version; IBS-QOL: Irritable bowel syndrome quality of life instrument; IBS: Irritable bowel syndrome; ICBT: Internet-based cognitive behavior therapy; MADRS-S: The montgomery asberg depression rating scale self report; SCL-SOM: Symptom checklist - somatization subscale; SDS: Sheehan disability scales; SSP-STA: Swedish Universites scales of personalities - somatic trait anxiety subscale; SSP-STA: Swedish Universites scales of personalities psychic trait anxiety subscale; VSI: Visceral sensitivity index.

\section{Competing interests}

The authors declare that they have no competing interests.

\section{Authors' contributions}

BL conceived the idea of the study, wrote the treatment manual, supervised the treatment, conducted the analyses, and drafted the manuscript. ErA interviewed the participants and drafted the manuscript. PL supervised the recruitment process. JML provided expertise in interpretation of the results. $K G, K M, J N$, and $K R$ collected outcome data and performed preliminary data analysis. KG and JN also treated participants and KM and KR also interviewed participants. EvA assisted in interpretation of the results and drafted the manuscript. TH supervised the preliminary data analysis. $\mathrm{HH}$ supervised the data analysis and drafted the manuscript. EH participated in the treatment design, co-authored the treatment manual and drafted the manuscript. All authors contributed to the manuscript and interpretation of the results and read and approved the final manuscript.

\section{Acknowledgements}

We would like to thank Gerhard Andersson, Nils Lindefors, and Christian Rück for supervision of the research group. We would also like to thank Lisa Falk and Amanda Wibron-Vesterlund who treated participants and Per Carlbring who assisted with the participant recruitment. 


\section{Author details}

'Department of Clinical Neuroscience, Division of Psychology, Karolinska Institutet, Nobels väg 9, Stockholm 171 65, Sweden. ${ }^{2}$ Department of Clinical Neuroscience, Division of Psychiatry, Karolinska Institutet, Stockholm, Sweden. ${ }^{3}$ Department of Gastroenterology, Sabbatsbergs Hospital, Stockholm, Sweden. ${ }^{4}$ Department of Medicine Huddinge, Karolinska Institutet, Stockholm, Sweden. ${ }^{5}$ Department of Medicine, University at Buffalo School of Medicine and Biomedical Science, ECMC, Buffalo, NY, USA. ${ }^{6}$ Department of Psychology, Uppsala University, Uppsala, Sweden. ${ }^{7}$ Department of Behavioural Sciences and Learning, Linköping University, Linköping, Sweden. ${ }^{8}$ Department of Clinical Neuroscience, Osher Center for Integrative Medicine, Karolinska Institutet, Stockholm, Sweden.

\section{Received: 12 June 2013 Accepted: 14 November 2013}

Published: 19 November 2013

\section{References}

1. Lovell RM, Ford AC: Global prevalence of and risk factors for irritable bowel syndrome: a meta-analysis. Clin Gastroenterol Hepatol 2012, 10:712-721. e4.

2. Longstreth G, Thompson W, Chey W, Houghton L, Mearin F, Spiller RC Functional bowel disorders. Gastroenterology 2006, 130:1480-1491.

3. Halder S, Locke G, Talley NJ, Fett S, Zinsmeister A, Melton L: Impact of functional gastrointestinal disorders on health-related quality of life: a population-based case-control study. Aliment Pharmacol Ther 2004, 19:233-242.

4. Talley NJ, Gabriel S, Harmsen W, Zinsmeister A, Evans R: Medical costs in community subjects with irritable bowel syndrome.

Gastroenterology 1995, 109:1736-1741.

5. Ford A, Talley NJ, Schoenfeld P, Quigley E, Moayyedi P: Efficacy of antidepressants and psychological therapies in irritable bowel syndrome: systematic review and meta-analysis. Gut 2009, 58:367-378.

6. Ljótsson $B$, Hesser $H$, Andersson E, Lindfors P, Hursti T, Rück C, Lindefors N, Andersson $G$, Hedman E: Mechanisms of change in an exposure-based treatment for irritable bowel syndrome. J Consult Clin Psychol 2013. http://dx.doi.org/10.1037/a0033439.

7. Kraemer H, Wilson G, Fairburn C, Agras W: Mediators and moderators of treatment effects in randomized clinical trials. Arch Gen Psychiatry 2002, 59:877-883.

8. Whitehead W, Palsson O, Jones K: Systematic review of the comorbidity of irritable bowel syndrome with other disorders: what are the causes and implications? Gastroenterology 2002, 122:1140-1156.

9. Drossman DA, Camilleri M, Mayer EA, Whitehead W: AGA technical review on irritable bowel syndrome. Gastroenterology 2002, 123:2108-2131.

10. Galovski T, Blanchard E: The treatment of irritable bowel syndrome with hypnotherapy. App/ Psychophysiol Biofeedback 1998, 23:219-232.

11. Guthrie E, Creed F, Dawson D, Tomenson B: A controlled trial of psychological treatment for the irritable bowel syndrome. Gastroenterology 1991, 100:450-457.

12. Carruthers HR, Morris J, Tarrier N, Whorwell PJ: Mood color choice helps to predict response to hypnotherapy in patients with irritable bowel syndrome. BMC Complement Altern Med 2010, 10:75

13. Reme SE, Kennedy T, Jones R, Darnley S, Chalder T: Predictors of treatment outcome after cognitive behavior therapy and antispasmodic treatment for patients with irritable bowel syndrome in primary care. J Psychosom Res 2010, 68:385-388.

14. Drossman DA, Toner B, Whitehead W, Diamant N, Dalton C, Duncan S, Emmott S, Proffitt V, Akman D, Frusciante K, Le T, Meyer K, Bradshaw B, Mikula K, Morris C, Blackman C, Hu Y, Jia H, Li J, Koch G, Bangdiwala S: Cognitive-behavioral therapy versus education and desipramine versus placebo for moderate to severe functional bowel disorders. Gastroenterology 2003, 125:19-31.

15. Blanchard EB, Lackner JM, Gusmano R, Gudleski GD, Sanders K, Keefer L, Krasner S: Prediction of treatment outcome among patients with irritable bowel syndrome treated with group cognitive therapy. Behav Res Ther 2006, 44:317-337.

16. Blanchard EB, Schwarz SP, Neff DF, Gerardi MA: Prediction of outcome from the self-regulatory treatment of irritable bowel syndrome. Behav Res Ther 1988, 26:187-190.

17. Blanchard E, Scharff L, Payne A, Schwarz S, Suls J, Malamood H: Prediction of outcome from cognitive-behavioral treatment of irritable bowel syndrome. Behav Res Ther 1992, 30:647-650.
18. Whorwell PJ, Prior A, Colgan SM: Hypnotherapy in severe irritable bowel syndrome: further experience. Gut 1987, 28:423-425.

19. Harvey R, Hinton R, Gunary R, Barry R: Individual and group hypnotherapy in treatment of refractory irritable bowel syndrome. Lancet 1989, 1:424-425.

20. Gonsalkorale W, Houghton L, Whorwell P: Hypnotherapy in irritable bowel syndrome: a large-scale audit of a clinical service with examination of factors influencing responsiveness. Am J Gastroenterol 2002, 97:954-961.

21. Creed F, Ratcliffe J, Fernandes L, Palmer S, Rigby C, Tomenson B, Guthrie E, Read N, Thompson D: Outcome in severe irritable bowel syndrome with and without accompanying depressive, panic and neurasthenic disorders. Br J Psychiatry 2005, 186:507-515.

22. Creed F, Guthrie E, Ratcliffe J, Fernandes L, Rigby C, Tomenson B, Read N, Thompson D: North of England I: does psychological treatment help only those patients with severe irritable bowel syndrome who also have a concurrent psychiatric disorder? Aust NZ J Psychiat 2005, 39:807-815.

23. Creed F, Tomenson B, Guthrie E, Ratcliffe J, Fernandes L, Read N, Palmer S, Thompson D: The relationship between somatisation and outcome in patients with severe irritable bowel syndrome. J Psychosom Res 2008, 64:613-620

24. Lackner JM, Jaccard J, Krasner SS, Katz LA, Gudleski GD, Blanchard EB: How does cognitive behavior therapy for irritable bowel syndrome work? a mediational analysis of a randomized clinical trial. Gastroenterology 2007 133:433-444.

25. Ljótsson B, Andersson G, Andersson E, Hedman E, Lindfors P, Andréewitch S, Rück C, Lindefors N: Acceptability, effectiveness, and cost-effectiveness of internet-based exposure treatment for irritable bowel syndrome in a clinical sample: a randomized controlled trial. BMC Gastroenterol 2011, 11:110.

26. Ljótsson B, Andréewitch S, Hedman E, Rück C, Andersson G, Lindefors N: Exposure and mindfulness based therapy for irritable bowel syndrome an open pilot study. J Behav Ther Exp Psychiatry 2010, 41:185-190.

27. Ljótsson B, Falk L, Vesterlund AW, Hedman E, Lindfors P, Rück C, Hursti T, Andréewitch S, Jansson L, Lindefors N, Andersson G: Internet-delivered exposure and mindfulness based therapy for irritable bowel syndrome-a randomized controlled trial. Behav Res Ther 2010, 48:531-539.

28. Ljótsson B, Hedman E, Andersson E, Hesser H, Lindfors P, Hursti T, Rydh S, Rück C, Lindefors N, Andersson G: Internet-delivered exposure-based treatment vs. stress management for irritable bowel syndrome: a randomized trial. Am J Gastroenterol 2011, 106:1481-1491.

29. Craske MG, Wolitzky-Taylor KB, Labus J, Wu S, Frese M, Mayer EA, Naliboff BD: A cognitive-behavioral treatment for irritable bowel syndrome using interoceptive exposure to visceral sensations. Behav Res Ther 2011, 49:413-421

30. Hunt M, Moshier S, Milonova M: Brief cognitive-behavioral internet therapy for irritable bowel syndrome. Behav Res Ther 2009, 47:797-802.

31. Labus J, Bolus R, Chang L, Wiklund I, Naesdal J, Mayer EA, Naliboff B: The visceral sensitivity index: development and validation of a gastrointestinal symptom-specific anxiety scale. Aliment Pharmacol Ther 2004, 20:89-97.

32. Jerndal $P$, Ringstrom $G$, Agerforz $P$, Karpefors $M$, Akkermans $L$, Bayati $A$ Simrén M: Gastrointestinal-specific anxiety: an important factor for severity of GI symptoms and quality of life in IBS. Neurogastroenterol Motil 2010, 22:646-e179

33. Labus J, Mayer EA, Chang L, Bolus R, Naliboff B: The central role of gastrointestinal-specific anxiety in irritable bowel syndrome: further validation of the visceral sensitivity index. Psychosom Med 2007, 69:89-98.

34. Ljótsson B, Hedman E, Lindfors P, Hursti T, Lindefors N, Andersson G, Rück C: Long-term follow-up of internet-delivered exposure and mindfulness based treatment for irritable bowel syndrome. Behav Res Ther 2011, 49:58-61.

35. Hedman E, Ljótsson B, Rück C, Furmark T, Carlbring P, Lindefors $N$ Andersson G: Internet administration of self-report measures commonly used in research on social anxiety disorder: a psychometric evaluation. Comput Hum Behav 2010, 26:736-740.

36. Wiklund I, Fullerton S, Hawkey C, Jones R, Longstreth G, Mayer EA Peacock R, Wilson I, Naesdal J: An irritable bowel syndrome-specific symptom questionnaire: development and validation. Scand $J$ Gastroenterol 2003, 38:947-954.

37. Sheehan D, Lecrubier Y, Sheehan K, Amorim P, Janavs J, Weiller E, Hergueta T, Baker R, Dunbar G: The Mini-International Neuropsychiatric Interview (M.I.N.I.): the development and validation of a structured diagnostic psychiatric interview for DSM-IV and ICD-10. J Clin Psychiatry 1998, 59(Suppl 20):22-33. 
38. Svanborg P, Åsberg M: A new self-rating scale for depression and anxiety states based on the comprehensive psychopathological rating scale. Acta Psychiatr Scand 1994, 89:21-28.

39. Gustavsson JP, Bergman H, Edman G, Ekselius L, Von Knorring L, Linder J: Swedish universities scales of personality (SSP): construction, internal consistency and normative data. Acta Psychiatr Scand 2000, 102:217-225.

40. Derogatis LR, Lipman RS, Rickels K, Uhlenhuth EH, Covi L: The Hopkins symptom checklist (HSCL) - A measure of primary symptom dimensions. In Psychological Measurements in Psychopharmacology. Edited by Pichot P. Basel: Karger; 1974

41. Rosenstiel A, Keefe F: The use of coping strategies in chronic low back pain patients: relationship to patient characteristics and current adjustment. Pain 1983, 17:33-44.

42. Reiss S, Peterson RA, Gursky DM, McNally RJ: Anxiety sensitivity, anxiety frequency and the prediction of fearfulness. Behav Res Ther 1986, 24:1-8,

43. Patrick D, Drossman DA, Frederick I, DiCesare J, Puder K: Quality of life in persons with irritable bowel syndrome: development and validation of a new measure. Dig Dis Sci 1998, 43:400-411.

44. Sheehan D: The Sheehan Disability Scales, The Anxiety Disease and How to Overcome It. New York City, NY: Charles Scribner and Sons; 1983:151.

45. Hedman E, Ljótsson B, Lindefors N: Cognitive behavior therapy via the Internet: a systematic review of applications, clinical efficacy and cost-effectiveness. Expert Rev Pharmacoecon Outcomes Res 2012, 12:745-764.

46. Steketee G, Chambless D: Methodological issues in prediction of treatment outcome. Clin Psychol Rev 1992, 12:387-400.

47. MacCallum RC, Zhang S, Preacher KJ, Rucker DD: On the practice of dichotomization of quantitative variables. Psychol Methods 2002, 7:19-40.

48. Clark D, Ehlers $A$ : An overview of the cognitive theory and treatment of panic disorder. App Prev Psychol 1993, 2:131-139.

49. Hayes SC, Wilson K, Gifford E, Follette V, Strosahl K: Experiential avoidance and behavioral disorders: a functional dimensional approach to diagnosis and treatment. J Consult Clin Psychol 1996, 64:1152-1168.

50. Rief W, Broadbent E: Explaining medically unexplained symptoms-models and mechanisms. Clin Psychol Rev 2007, 27:821-841.

51. Dorn SD, Kaptchuk TJ BPJ, Nguyen LT, Canenguez K, Nam BH, Woods KB, Conboy LA, Stason WB, Lembo AJ: A meta-analysis of the placebo response in complementary and alternative medicine trials of irritable bowel syndrome. Neurogastroenterol Motil 2007, 19:630-637.

52. Spiller RC, Aziz Q, Creed F, Emmanuel A, Houghton L, Hungin P, Jones R, Kumar D, Rubin G, Trudgill N, Whorwell P: Guidelines on the irritable bowel syndrome: mechanisms and practical management. Gut 2007, 56:1770-1798.

53. Reme SE, Darnley S, Kennedy T, Chalder T: The development of the irritable bowel syndrome-behavioral responses questionnaire. J Psychosom Res 2010, 69:319-325.

54. Kennedy T, Jones R, Darnley S, Seed P, Wessely S, Chalder T: Cognitive behaviour therapy in addition to antispasmodic treatment for irritable bowel syndrome in primary care: randomised controlled trial. Br Med J 2005, 331:435

55. Hedman E, Andersson E, Ljótsson B, Andersson G, Andersson E, Schalling M, Lindefors N, Rück C: Clinical and genetic outcome determinants of Internet- and group-based cognitive behavior therapy for social anxiety disorder. Acta Psychiatr Scand 2012, 126:126-136.

56. Hedman E, Lindefors $N$, Andersson $G$, Andersson E, Lekander M, Rück C, Ljótsson B: Predictors of outcome in Internet-based cognitive behavior therapy for severe health anxiety. Behav Res Ther 2013, 51:711-717.

57. Ljótsson B, Atterlöf E, Lagerlöf M, Andersson E, Jernelöv S, Hedman E, Kemani M, Wicksell RK: Internet-delivered acceptance and values-based exposure treatment for fibromyalgia: a pilot study. Cogn Behav Ther 2013. http://dx.doi.org/10.1080/16506073.2013.846401

58. Lackner JM, Gudleski GD, Keefer L, Krasner SS, Powell C, Katz LA: Rapid response to cognitive behavior therapy predicts treatment outcome in patients with irritable bowel syndrome. Clin Gastroenterol Hepatol 2010, 8:426-432.

doi:10.1186/1471-230X-13-160

Cite this article as: Ljótsson et al:: Prediction of symptomatic improvement after exposure-based treatment for irritable bowel syndrome. BMC Gastroenterology 2013 13:160.

\section{Submit your next manuscript to BioMed Central and take full advantage of:}

- Convenient online submission

- Thorough peer review

- No space constraints or color figure charges

- Immediate publication on acceptance

- Inclusion in PubMed, CAS, Scopus and Google Scholar

- Research which is freely available for redistribution

Submit your manuscript at www.biomedcentral.com/submit
() Biomed Central 